\title{
Evaluation of Small Interfering RNA Delivery into Cells by Reverse Transfection in Suspension with Cationic Liposomes
}

\author{
Yoshiyuki Hattori $^{*}$, Yuki Yoshiike ${ }^{1}$, Maho Honda ${ }^{2}$, Hiroaki Ohno ${ }^{2}$, Hiraku Onishi ${ }^{1}$ \\ ${ }^{1}$ Department of Drug Delivery Research, Hoshi University, Shinagawa, Japan \\ ${ }^{2}$ Graduate School of Pharmaceutical Sciences, Kyoto University, Kyoto, Japan \\ Email: *yhattori@hoshi.ac.jp
}

How to cite this paper: Hattori, Y., Yoshiike, Y., Honda, M., Ohno, H. and Onishi, H. (2017) Evaluation of Small Interfering RNA Delivery into Cells by Reverse Transfection in Suspension with Cationic Liposomes. Pharmacology \& Pharmacy, 8, 129-139.

https://doi.org/10.4236/pp.2017.85009

Received: April 5, 2017

Accepted: May 12, 2017

Published: May 15, 2017

Copyright $\odot 2017$ by authors and Scientific Research Publishing Inc. This work is licensed under the Creative Commons Attribution International License (CC BY 4.0).

http://creativecommons.org/licenses/by/4.0/

(c) (i) Open Access

\begin{abstract}
Successful gene silencing by small interfering RNA (siRNA) requires efficient uptake of siRNA into targeted cells. For in vitro transfection of siRNA using cationic liposomes, two types of transfection method are currently being used: conventional (forward; Fw) and reverse (Rev) transfections. Here, to investigate an efficient siRNA transfection method using cationic liposomes, we compared the transfection efficiency of siRNA between Fw-transfection and Rev-transfection methods with various types of cationic liposomes. In Fwtransfection, siRNA/cationic liposomes complex (siRNA lipoplexes) was added to pre-plated cells. In contrast, Rev-transfection was performed by co-incubation of cells with siRNA lipoplexes in suspension. As a result, Rev-transfection with 1,2-dioleoyl-3-trimethylammonium-propane (DOTAP)-based or cationic cholesterol derivative-based liposomes could deliver siRNA into the cells via efficient cellular association, and induce an improved gene silencing effect by siRNA compared with Fw-transfection. Furthermore, Rev-transfection did not show increased cytotoxicity compared with Fw-transfection. These findings suggested that Rev-transfection in suspension has better potential for efficient transfection of siRNA into cells with minimal toxicity.
\end{abstract}

\section{Keywords}

Cationic Liposome, siRNA Delivery, Reverse Transfection, Tumor Cells

\section{Introduction}

Synthetic small interfering RNAs (siRNAs), which are small, double-stranded RNAs, are substrates for the RNA-induced silencing complex [1]. Successful gene silencing by siRNA requires efficient uptake of siRNA into targeted cells. 
For in vitro experiments, cationic liposomes have often been used for siRNA transfection into the cells [2] [3]. In siRNA transfection by cationic liposomes, two types of transfection methods are currently being used: conventional (forward; Fw) and reverse (Rev) transfections. They differ in the order and timing of the addition of the three necessary components of transfection: siRNA, cationic liposomes, and cells. In the Fw-transfection method, siRNA and cationic liposomes are complexed and then the siRNA lipoplexes are added to pre-plated cells. In the Rev-transfection method, all three components are added to the wells essentially at the same time. Compared with Fw-transfection, Rev-transfection enables direct and sufficient contact between cells and siRNA lipoplexes, thereby improving transfection efficiency [4]. In particular, solid-phase Rev-transfection, also known as surface-mediated transfection, is used as a high-throughput method for the parallel transfection of cells on microarrays [5] [6] or microplates [7]. In solid-phase Rev-transfection, siRNA complexes using cationic liposomes such as commercially available Lipofectamine 2000 (Invitrogen, Carlsbad, CA, USA) and Lipofectamine RNAiMax (Invitrogen) are attached to the bottom of cell culture plates by freeze-drying [4] [8] [9], and cells take up these complexes after plating on the culture plate. However, solid-phase Rev-transfection has been restricted so far to microarray- or microplate-based analyses. Reverse transfection can be also performed by co-incubation of cells with siRNA lipoplexes in suspension (Rev-transfection in suspension). Previously, it has been reported that transfection activities by commercially available Lipofectamine 2000 and RNAiFect (Qiagen, Hilden, Germany) could be improved by mixing siRNA complexes with cells in suspension and allowing the cells to attach in the presence of the complex [10]. However, to the best of our knowledge, there are still few reports on the application of Rev-transfection in suspension with cationic liposomes for the delivery of siRNA into cells. Therefore, in this study, we investigated Rev-transfection of siRNA in suspension with various type of cationic liposomes, and demonstrated that Rev-transfection in suspension with 1,2-dioleoyl-3-trimethylammonium-propane (DOTAP)-based or cationic cholesterol-derivative based liposomes could increase gene silencing activity in the cells via efficient cellular association.

\section{Materials and Methods}

\subsection{Materials}

1,2-Dioleoyl-3-trimethylammonium-propane (DOTAP) methyl sulfate salt (Figure 1) was obtained from Avanti Polar Lipids Inc. (Alabaster, AL, USA). Cholesterol (Chol) was purchased from Wako Pure Chemical Industries, Ltd. (Osaka, Japan). $\mathrm{N}$-(2-(2-Hydroxyethylamino)ethyl)cholesteryl-3-carboxamide (OH-Chol) (Figure 1) and cholesteryl (2-((2-hydroxyethyl)amino)ethyl)carbamate (OH-C-Chol) (Figure 1) were synthesized as described previously [11]. 1,2-Dioleoyl-sn-glycero-3-phosphoethanolamine (DOPE) was obtained from NOF Co. Ltd. (Tokyo, Japan). All other chemicals were of the finest grade available. 



Figure 1. Structure of cationic lipids used in this study: 1,2-dioleoyl-3-trimethylammonium-propane (DOTAP) methyl sulfate salt, (3S)- $N$-(2-(2-hydroxyethylamino)ethyl)cholesteryl-3-carboxamide (OH-Chol) and cholesteryl (2-((2-hydroxyethyl)amino)ethyl)carbamate (OH-C-Chol).

\subsection{Small Interfering RNAs}

siRNAs targeting nucleotides of firefly pGL3 luciferase (Luc siRNA) and nonsilencing siRNA (control [Cont] siRNA) as a negative control were synthesized by Sigma Genosys (Tokyo, Japan). The siRNA sequences of the Luc siRNA were: sense strand: 5'-GUGGAUUUCGAGUCGUCUUAA-3' and antisense strand: 5'-AAGACGACUCGAAAUCCACAU-3'. The siRNA sequences of the Cont siRNA as a negative control for Luc siRNA were: sense strand: 5'GUACCGCA CGUCAUUCGUAUC-3' ' and antisense strand: 5'-UACGAAUGACGUGCGGU ACGU-3'. Alexa Fluor 488-labeled AllStars Negative Control siRNA (AF-siRNA) was obtained from Qiagen (Valencia, CA, USA).

\subsection{Preparation of Cationic Liposomes and siRNA Lipoplexes}

Cationic liposomes were prepared from DOTAP/Chol or DOTAP/DOPE at a molar ratio of 1:1 using a thin-film hydration method, as reported previously [12]. Cationic cholesterol derivative-based liposomes were prepared from $\mathrm{OH}-$ Chol/DOPE or OH-C-Chol/DOPE at a molar ratio of 3:2 using a thin-film hydration method [11]. The thin film was hydrated with water at $60^{\circ} \mathrm{C}$ by vortex mixing for $1 \mathrm{~min}$. The liposomes were sonicated in a bath-type sonicator for 10 $\min$.

To prepare complexes of siRNA/cationic liposome (siRNA lipoplexes), each liposome preparation was added to 50 pmol siRNA at a charge ratio (+:-) of 4:1 of cationic lipid to siRNA for DOTAP/Chol and DOTAP/DOPE liposomes or 7:1 for OH-Chol/DOPE and OH-C-Chol/DOPE liposomes with gentle shaking and left at room temperature for $15 \mathrm{~min}$. The charge ratio (+:-) of liposomes: siRNA is expressed as the molar ratio of cationic lipid to siRNA phosphate.

\subsection{Size and ろ-Potential of Cationic Liposomes and siRNA Lipoplexes}

The particle size distributions of cationic liposomes and lipoplexes were meas- 
ured by the cumulant method using a light-scattering photometer (ELS-Z2, Otsuka Electronics Co., Ltd., Osaka, Japan) at $25^{\circ} \mathrm{C}$ after diluting the dispersion with an appropriate volume with water. The $\zeta$-potentials were measured using the ELS-Z2 at $25^{\circ} \mathrm{C}$ after diluting the dispersion with an appropriate volume of water.

\subsection{Cell Culture}

Human breast cancer MCF-7-Luc (TamR-Luc\#1) cells stably expressing firefly luciferase (pGL3) were donated by Dr. Kazuhiro Ikeda (Division of Gene Regulation and Signal Transduction, Research Center for Genomic Medicine, Saitama Medical University, Saitama, Japan). The cells were cultured in Dulbecco's modified Eagle's medium (DMEM), supplemented with $10 \%$ heat-inactivated fetal bovine serum (FBS), $100 \mu \mathrm{g} / \mathrm{mL}$ kanamycin, and $0.5 \mathrm{mg} / \mathrm{mL}$ G418 at $37^{\circ} \mathrm{C}$ in a $5 \% \mathrm{CO}_{2}$ humidified atmosphere.

\subsection{Transfection}

For Fw-transfection, MCF-7-Luc cells were detached with TrypLE ${ }^{\mathrm{TM}}$ Express (Invitrogen), plated into 6-well $(35 \mathrm{~mm})$ culture dishes at a density of $2.5 \times 10^{5}$ cells per well and then incubated for $1 \mathrm{~h}$ at $37^{\circ} \mathrm{C}$ in a $5 \% \mathrm{CO}_{2}$ humidified atmosphere. Each lipoplex with 50 pmol siRNA was diluted in $1 \mathrm{~mL}$ of DMEM (50 nM siRNA) supplemented with $10 \%$ FBS, and then added to the cells (Figure 2).

For Rev-transfection, MCF-7-Luc cells were detached with TrypLE ${ }^{\mathrm{TM}}$ Express, and $2.5 \times 10^{5}$ cells were suspended in $0.5 \mathrm{~mL}$ of DMEM supplemented with $10 \%$ FBS (Figure 2). Each lipoplex with 50 pmol siRNA was diluted in $0.5 \mathrm{~mL}$ of DMEM supplemented with $10 \%$ FBS, and then added to the cells suspended in $0.5 \mathrm{~mL}$ of the medium. The mixtures ( $1 \mathrm{~mL}$ ) of siRNA lipoplexes (50 nM siRNA) and suspended cells were transferred into 6-well culture dishes.

\subsection{Luciferase Activity}

Each lipoplex with 50 pmol Luc siRNA or Cont siRNA was transfected by the Fw-transfection or Rev-transfection methods into MCF-7-Luc cells in 6-well culture dishes (final $50 \mathrm{nM}$ siRNA concentration). Forty-eight hours after transfection, luciferase activity was measured as counts per sec (cps)/ $\mu \mathrm{g}$ protein using a luciferase assay system (Pica Gene, Toyo Ink Mfg. Co. Ltd., Tokyo, Japan) and BCA reagent (Pierce, Rockford, IL, USA), as reported previously [13]. Luciferase

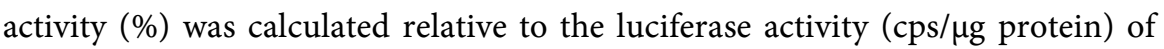
untransfected cells.

\subsection{Flow Cytometric Analysis}

For Fw-transfection, MCF-7-Luc cells were detached with TrypLE ${ }^{\mathrm{TM}}$ Express, plated into 6-well culture dishes at a density of $5.0 \times 10^{5}$ cells per well and then incubated for $1 \mathrm{~h}$. Each lipoplex with 50 pmol AF-siRNA was diluted in $1 \mathrm{~mL}$ of medium (50 nM siRNA) supplemented with 10\% FBS, and then added to the cells. For Rev-transfection, each lipoplex with 50 pmol AF-siRNA was diluted in 
(a) Fw-transfection


Figure 2. Schematic diagram of the reverse transfection (Rev-transfection) method with siRNA lipoplexes. In conventional transfection (Forward (Fw)-transfection), siRNA/cationic liposome complexes (siRNA lipoplexes) are diluted in culture medium, and then added to the pre-plated adherent cells (a). In Revtransfection, siRNA lipoplexes are diluted in culture medium, and are then mixed with an equal volume of medium-diluted cells, followed by transfer to a culture dish (b). Scale bar $=1,000 \mu \mathrm{m}$.

$0.5 \mathrm{~mL}$ of medium supplemented with $10 \% \mathrm{FBS}$, and then added to $5.0 \times 10^{5}$ cells suspended in $0.5 \mathrm{~mL}$ of medium. The mixtures $(1 \mathrm{~mL})$ of siRNA lipoplex (50 nM AF-siRNA) and suspended cells were transferred into 6-well culture dishes. After $3 \mathrm{~h}$ incubation, the cells were washed twice with $1 \mathrm{~mL}$ phosphatebuffered saline (PBS) to remove any unbound lipoplexes. The amount of AFsiRNA in the cells was determined by examining fluorescence intensity using a FACSCalibur flow cytometer (Becton Dickinson, San Jose, CA, USA), as described previously [14].

\subsection{Cytotoxicity}

For Fw-transfection, MCF-7-Luc cells were plated into 96-well culture dishes at a density of $2.5 \times 10^{4}$ cells per well and incubated for $1 \mathrm{~h}$. Each lipoplex with 50 pmol Cont siRNA was diluted in $1 \mathrm{~mL}$ of medium supplemented with $10 \% \mathrm{FBS}$, and then the mixture $(100 \mu \mathrm{L}$ ) was added to the cells (final $50 \mathrm{nM}$ siRNA concentration).

For Rev-transfection, each lipoplex with 50 pmol Cont siRNA was diluted in $0.5 \mathrm{~mL}$ of medium supplemented with $10 \% \mathrm{FBS}$, and then added to $2.5 \times 10^{5}$ 
cells suspended in $0.5 \mathrm{~mL}$ of the medium. The mixtures $(100 \mu \mathrm{L})$ of the lipoplexes and $2.5 \times 10^{4}$ cells were transferred into 96-well culture dishes (final $50 \mathrm{nM}$ at siRNA concentration).

After a $24 \mathrm{~h}$ incubation period, cell numbers were determined using a Cell Counting Kit-8 (Dojindo Laboratories, Kumamoto, Japan). Cell viability was expressed as relative to the absorbance at $450 \mathrm{~nm}$ of untransfected cells.

\subsection{Statistical Analysis}

Data were compared using analysis of variance and evaluated with Student's $t$ test. A $p$ value of 0.05 or less was considered significant.

\section{Results and Discussion}

First, we prepared DOTAP-based and cationic cholesterol derivative-based liposomes for siRNA delivery by $\mathrm{Fw}$ - and Rev-transfections, respectively. In this study, we used DOTAP, OH-Chol, or OH-C-Chol (Figure 1) as a cationic lipid, and DOPE or Chol as a neutral lipid. Because we reported previously that cationic liposomes composed of OH-Chol or OH-C-Chol and DOPE could efficiently deliver siRNA into tumor cells [11] [14]. In the formulations with DOTAPbased liposomes, cationic liposomes composed of DOTAP and cholesterol (Chol) or DOPE were prepared, because their formulations were often used for siRNA delivery [12] [15] [16]. The sizes of DOTAP/Chol, DOTAP/DOPE, OH-Chol/ DOPE, and OH-C-Chol/DOPE liposomes were approximately $100-130 \mathrm{~nm}$, and their $\zeta$-potentials were $47-56 \mathrm{mV}$ (Table 1). When the lipoplexes were prepared with siRNA, their sizes were approximately $190-220 \mathrm{~nm}$ and their $\zeta$-potentials were about $42-47 \mathrm{mV}$.

Next, we examined the effect of transfection methods on the gene knockdown effect by the lipoplexes with $50 \mathrm{nM}$ siRNA using a luciferase assay system with MCF-7-Luc cells. Conventional transfection (Fw-transfection) is a common technique for delivering siRNA into cells for gene silencing (Figure 2). In this standard approach, siRNA lipoplexes are added to adherent cells on the surface of culture plate. Generally, siRNA lipoplexes are added into cells plated $24 \mathrm{~h}$ prior to transfection; however, in this study, siRNA lipoplexes were added into the cells plated $1 \mathrm{~h}$ prior to transfection, because the number of cells between

Table 1. Formulae, size, and $\zeta$-potentials of cationic liposomes and siRNA lipoplexes.

\begin{tabular}{lccccc}
\hline \multirow{2}{*}{ Formulation } & \multirow{2}{*}{$\begin{array}{c}\text { Molar } \\
\text { ratio }\end{array}$} & \multicolumn{2}{c}{ Liposome $^{\mathrm{a})}$} & \multicolumn{2}{c}{ Lipoplex of siRNA $^{\mathrm{a}, \mathrm{b})}$} \\
\cline { 3 - 6 } & & Size $(\mathrm{nm})$ & $\zeta$-potential $(\mathrm{mV})$ & Size $(\mathrm{nm})$ & $\zeta$-potential $(\mathrm{mV})$ \\
\hline DOTAP/Chol & $1 / 1$ & $101.2 \pm 2.5$ & $56.0 \pm 1.0$ & $189.5 \pm 5.0$ & $41.7 \pm 0.4$ \\
DOTAP/DOPE & $1 / 1$ & $116.2 \pm 2.9$ & $49.7 \pm 0.6$ & $189.0 \pm 6.0$ & $44.4 \pm 0.8$ \\
OH-Chol/DOPE & $3 / 2$ & $126.0 \pm 2.9$ & $52.8 \pm 0.9$ & $220.1 \pm 1.9$ & $46.7 \pm 0.8$ \\
OH-C-Chol/DOPE & $3 / 2$ & $120.5 \pm 2.7$ & $47.5 \pm 1.8$ & $195.3 \pm 5.2$ & $46.1 \pm 1.8$
\end{tabular}

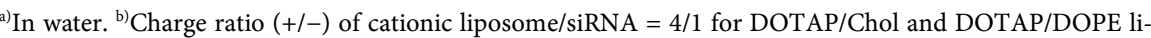
poplexes, and 7/1 for $\mathrm{OH}-\mathrm{Chol} / \mathrm{DOPE}$ and $\mathrm{OH}-\mathrm{C}-\mathrm{Chol}$ lipoplexes, respectively. Values represent means \pm S.D. $(\mathrm{n}=3)$. 
Fw-transfection and Rev-transfection must be uniform. In contrast, in Revtransfection, siRNA lipoplexes were diluted in culture medium, and mixed with an equal volume of medium diluted with MCF-7 cells (Figure 2).

For DOTAP-based formulations, DOTAP/Chol lipoplexes with Luc siRNA did not suppress luciferase activity by Fw-transfection; however, moderate suppression of luciferase activity was observed by Rev-transfection (Figure 3(a)). In contrast, DOTAP/DOPE lipoplexes with Luc siRNA significantly suppressed luciferase activity by Fw-transfection, and its gene silencing effect was increased by Rev-transfection (Figure 3(a)). For cationic cholesterol derivative-based formulations, $\mathrm{OH}-\mathrm{Chol} / \mathrm{DOPE}$ and $\mathrm{OH}-\mathrm{C}-\mathrm{Chol} / \mathrm{DOPE}$ lipoplexes could strongly suppress luciferase activities by both Fw-transfection and Rev-transfection methods, respectively, although Rev-transfection slightly improved gene silencing activities compared with Fw-transfection (Figure 3(b)). From the results of these gene silencing effects, Rev-transfection in suspension with siRNA lipoplexes could improve gene knockdown by siRNA.

DOTAP/DOPE lipoplexes exhibited higher gene silencing activity than DOTAP/Chol lipoplexes (Figure 3(a)). It is generally known that cationic lipoplexes need to escape from endosomes after cellular internalization for the induction of gene knockdown. DOPE has been widely used as a co-lipid for cationic liposome-mediated transfection of siRNA and plasmid DNA [17]. The role of DOPE is not fully understood, but it might affect the structural transition of cationic liposomes at acidic pH in late endosomes in cells [18] [19], which promotes endosomal escape of siRNA lipoplexes. From these findings, DOTAP/ DOPE lipoplexes might be able to induce high gene silencing effects in the cells compared with DOTAP/Chol lipoplexes.
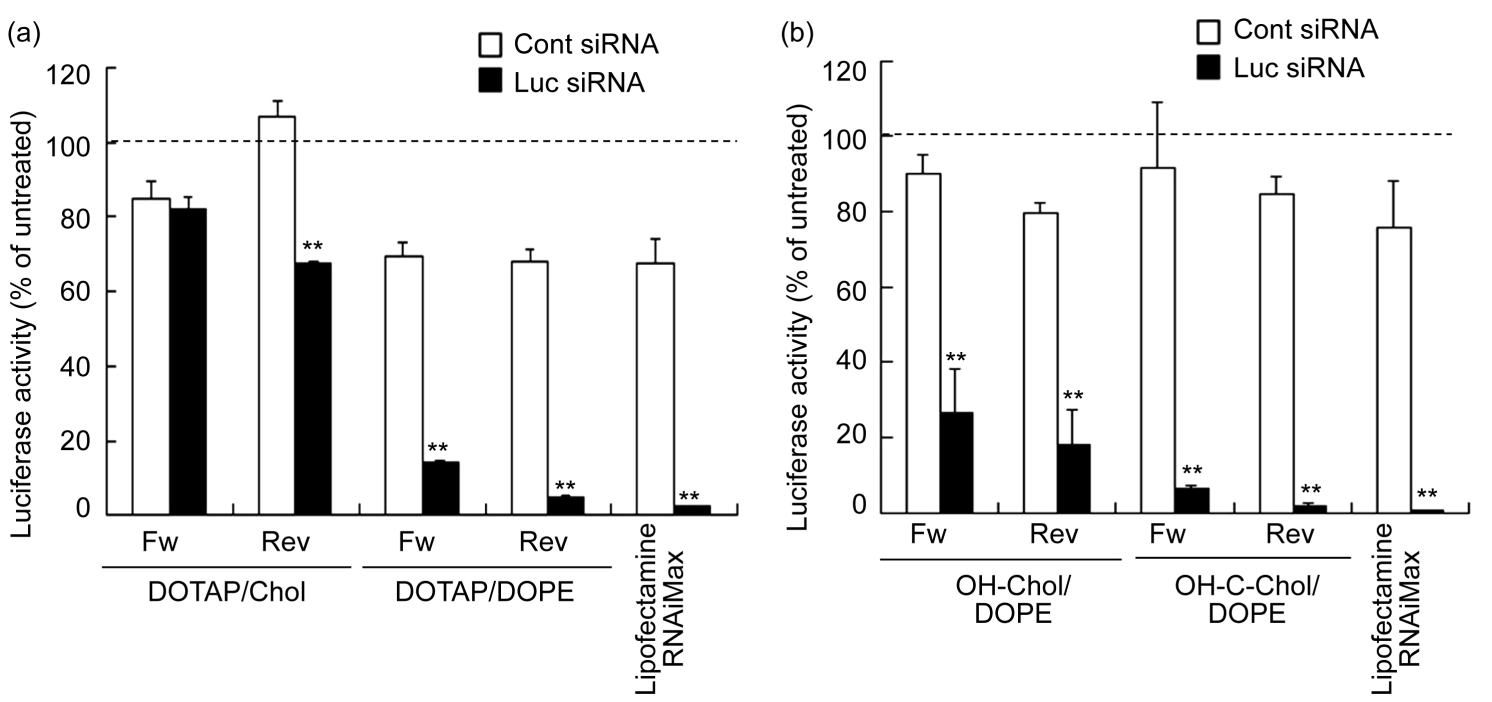

Figure 3. Effect of transfection method on gene silencing activity in MCF-7-Luc cells $48 \mathrm{~h}$ after transfection. (a) DOTAP/Chol and DOTAP/DOPE lipoplexes were transfected into the cells using the Fw-transfection or Revtransfection methods. (b) OH-Chol/DOPE and OH-C-Chol/DOPE lipoplexes were transfected into the cells by Fw-transfection or Rev-transfection methods. These lipoplexes were added to cells with $50 \mathrm{nM}$ small interfering RNA (siRNA). Commercially available transfection reagent Lipofectamine RNAiMax was used as a control. Each column represents the mean \pm S.D. $(n=3)$. ${ }^{* *} p<0.01$ compared with control (Cont) siRNA. 
To clarify the relationship between the gene silencing effect and cellular uptake in Fw-transfection and Rev-transfection with siRNA lipoplexes, we examined the cellular association of lipoplexes by flow cytometric analysis. In Fwtransfection, DOTAP/Chol lipoplexes exhibited higher cellular association than DOTAP/DOPE lipoplexes (Figure 4(a) and Figure 4(b)), indicating that the inclusion of cholesterol in a formulation with cationic liposomes resulted in good uptake by the cells, but could not induce a gene silencing effect. Furthermore, cellular associations with DOTAP/Chol (Figure 4(a)), DOTAP/DOPE (Figure 4(b)), OH-Chol/DOPE (Figure 4(c)), and OH-C-Chol/DOPE (Figure 4(d)) lipoplexes after Rev-transfection were higher than those after Fw-transfection, respectively (Figure 4(e)). This result corresponded to those of gene knockdown efficacy (Figure 3). From the results, Rev-transfection with siRNA lipoplexes could deliver siRNA into the cells via efficient cellular association and induce a large gene silencing effect using siRNAs.

Finally, we investigated cytotoxicity after Fw- and Rev-transfections with siRNA lipoplexes. As a result, in any siRNA lipoplexes, Rev-transfection did not


Figure 4. Cellular association at $3 \mathrm{~h}$ after Fw-transfection or Rev-transfection of siRNA lipoplexes. (a) DOTAP/Chol lipoplexes, (b) DOTAP/DOPE lipoplexes, (c) OH-Chol/DOPE lipoplexes, and (d) OH-C-Chol/DOPE lipoplexes were formed by mixing with Alexa Fluor ${ }^{\varpi} 488$-labeled small interfering RNA (AF-siRNA). These lipoplexes were added to cells with a final concentration of 50 nM siRNA. The association of siRNA lipoplexes with MCF-7 cells was determined on the basis of Alexa Fluor ${ }^{\circledR} 488$-fluorescence by flow cytometry. (e) Each column represents the mean fluorescent intensity \pm S.D. $(n=3)$. 
strongly increase cytotoxicity compared with Fw-transfection (Figure 5). These findings suggested that the Rev-transfection method has potential for efficient transfection of siRNA into cells with minimal toxicity.

In solid-phase Rev-transfection, the siRNA complex must be freeze-dried on a culture plate in small volumes. The one of advantages for Rev-transfection in suspension is the ability to easily prepare a large number of plates in larger volumes. However, the reasons for the improvements in gene silencing (Figure 3) and cellular uptake (Figure 4) observed at the Rev-transfection in suspension are not clear. Amarzguioui described that in Rev-transfection in suspension, a more favorable cell surface environment for the attachment of siRNA lipoplexes might be generated after partial removal of surface proteins by treatment with trypsin [10]. However, in our study, Fw-transfection was performed with cells treated with TrypLE ${ }^{\mathrm{TM}}$ Express for detachment and plated $1 \mathrm{~h}$ prior to transfection. TrypLE ${ }^{\mathrm{TM}}$ Express is an enzyme that cleaves peptide bonds on the C-terminal sides of lysine and arginine as well as trypsin. This indicated that treatment of the cells with trypsin was not a critical factor for the improvement of transfection efficiency by the Rev-transfection method. We speculated that transfection efficiency by Rev-transfection might be improved through an increased area of cellular surface that could associate with siRNA lipoplexes in suspension. Alternatively, morphological changes associated with attachment to the surface of the culture plate might affect cellular uptake of siRNA lipoplexes.

In conclusion, Rev-transfection with siRNA lipoplexes in suspension could deliver siRNA into the cells via efficient cellular association and induce an improved gene silencing effect by siRNA compared with Fw-transfection. These findings suggested that the Rev-transfection method in suspension has potential for efficient transfection of siRNA into cells with minimal toxicity.

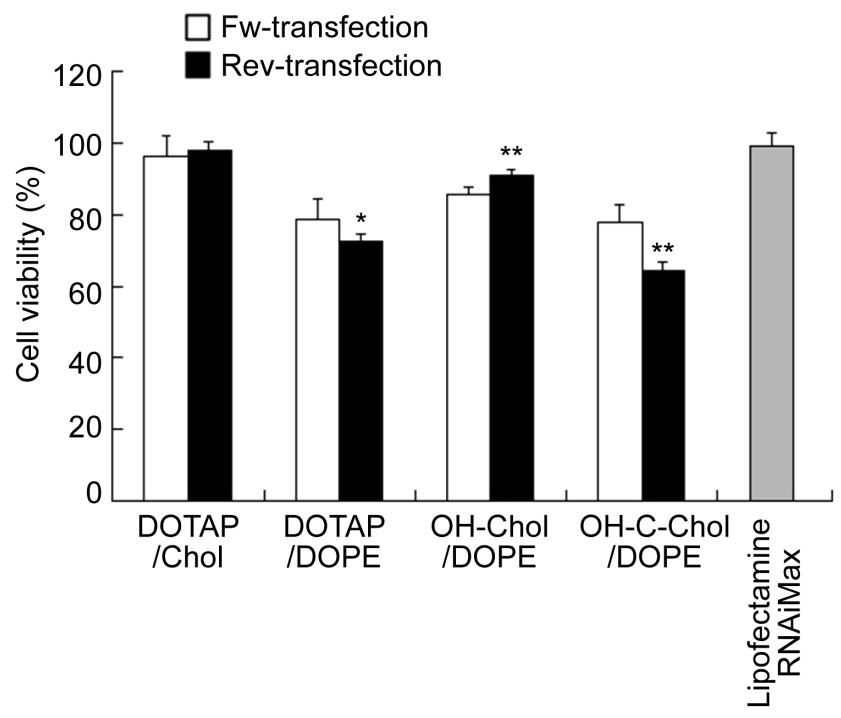

Figure 5. Cell viability $24 \mathrm{~h}$ after Fw-transfection or Rev-transfection with siRNA lipoplexes into MCF-7 cells. Each column represents the mean \pm S.D. $(n=6)$. Lipofectamine RNAiMax was used as a control. ${ }^{*} p<0.05,{ }^{* *} p<0.01$ compared with Fw-transfection. 


\section{Acknowledgement}

We declare no conflict of interests. This project was supported in part by a Grant-in-Aid for Scientific Research (C) from the Japan Society for the Promotion of Science (KAKENHI Grant Number 26460046).

\section{References}

[1] Kim, D.H., Behlke, M.A., Rose, S.D., Chang, M.S., Choi, S. and Rossi, J.J. (2005) Synthetic dsRNA Dicer Substrates Enhance RNAi Potency and Efficacy. Nature Biotechnology, 23, 222-226. https://doi.org/10.1038/nbt1051

[2] Zhang, S., Zhi, D. and Huang, L. (2012) Lipid-Based Vectors for siRNA Delivery. Journal of Drug Targeting, 20, 724-735. https://doi.org/10.3109/1061186X.2012.719232

[3] Ozpolat, B., Sood, A.K. and Lopez-Berestein, G. (2014) Liposomal siRNA Nanocarriers for Cancer Therapy. Advanced Drug Delivery Reviews, 66, 110-116.

[4] Erfle, H., Neumann, B., Liebel, U., Rogers, P., Held, M., Walter, T., Ellenberg, J. and Pepperkok, R. (2007) Reverse Transfection on Cell Arrays for High Content Screening Microscopy. Nature Protocols, 2, 392-329. https://doi.org/10.1038/nprot.2006.483

[5] Ziauddin, J. and Sabatini, D.M. (2001) Microarrays of Cells Expressing Defined cDNAs. Nature, 411, 107-110. https://doi.org/10.1038/35075114

[6] Mousses, S., Caplen, N.J., Cornelison, R., Weaver, D., Basik, M., Hautaniemi, S., Elkahloun, A.G., Lotufo, R.A., Choudary, A., Dougherty, E.R., Suh, E. and Kallioniemi, O. (2003) RNAi Microarray Analysis in Cultured Mammalian Cells. Genome Research, 13, 2341-2347. https://doi.org/10.1101/gr.1478703

[7] Erfle, H., Neumann, B., Rogers, P., Bulkescher, J., Ellenberg, J. and Pepperkok, R. (2008) Work Flow for Multiplexing siRNA Assays by Solid-Phase Reverse Transfection in Multiwell Plates. Journal of Biomolecular Screening, 13, 575-580. https://doi.org/10.1177/1087057108320133

[8] Wu, K., Xu, J., Liu, M., Song, W., Yan, J., Gao, S., Zhao, L. and Zhang, Y. (2013) Induction of Osteogenic Differentiation of Stem Cells via a Lyophilized microRNA Reverse Transfection Formulation on a Tissue Culture Plate. International Journal of Nanomedicine, 8, 1595-1607.

[9] Fujita, S., Ota, E., Sasaki, C., Takano, K., Miyake, M. and Miyake, J. (2007) Highly Efficient Reverse Transfection with siRNA in Multiple Wells of Microtiter Plates. Journal of Bioscience and Bioengineering, 104, 329-333. https://doi.org/10.1263/jbb.104.329

[10] Amarzguioui, M. (2004) Improved siRNA-Mediated Silencing in Refractory Adherent Cell Lines by Detachment and Transfection in Suspension. Biotechniques, 36, 766-778.

[11] Hattori, Y., Hara, E., Shingu, Y., Minamiguchi, D., Nakamura, A., Arai, S., Ohno, H., Kawano, K., Fujii, N. and Yonemochi, E. (2015) siRNA Delivery into Tumor Cells by Cationic Cholesterol Derivative-Based Nanoparticles and Liposomes. Biological and Pharmaceutical Bulletin, 38, 30-38. https://doi.org/10.1248/bpb.b14-00526

[12] Hattori, Y., Nakamura, A., Arai, S., Kawano, K., Maitani, Y. and Yonemochi, E. (2015) siRNA Delivery to Lung-Metastasized Tumor by Systemic Injection with Cationic Liposomes. Journal of Liposome Research, 25, 279-286. https://doi.org/10.3109/08982104.2014.992024

[13] Hattori, Y. and Maitani, Y. (2005) Folate-Linked Nanoparticle-Mediated Suicide Gene Therapy in Human Prostate Cancer and Nasopharyngeal Cancer with Herpes Simplex Virus Thymidine Kinase. Cancer Gene Therapy, 12, 796-809. 
https://doi.org/10.1038/sj.cgt.7700844

[14] Hattori, Y., Machida, Y., Honda, M., Takeuchi, N., Yoshiike, Y., Ohno, H. and Onishi, H. (2017) Small Interfering RNA Delivery into the Liver by Cationic Cholesterol Derivative-Based Liposomes. Journal of Liposome Research, 1-10.

[15] Resnier, P., LeQuinio, P., Lautram, N., Andre, E., Gaillard, C., Bastiat, G., Benoit, J. P. and Passirani, C. (2014) Efficient in Vitro Gene Therapy with PEG siRNA Lipid Nanocapsules for Passive Targeting Strategy in Melanoma. Biotechnology Journal, 9, 1389-1401. https://doi.org/10.1002/biot.201400162

[16] Lazebnik, M., Keswani, R.K. and Pack, D.W. (2016) Endocytic Transport of Polyplex and Lipoplex siRNA Vectors in HeLa Cells. Pharmaceutical Research, 33, 29993011. https://doi.org/10.1007/s11095-016-2022-1

[17] Aissaoui, A., Chami, M., Hussein, M. and Miller, A.D. (2011) Efficient Topical Delivery of Plasmid DNA to Lung in Vivo Mediated by Putative Triggered, PEGylated pDNA Nanoparticles. Journal of Controlled Release, 154, 275-284.

[18] Mochizuki, S., Kanegae, N., Nishina, K., Kamikawa, Y., Koiwai, K., Masunaga, H. and Sakurai, K. (2013) The Role of the Helper Lipid Dioleoylphosphatidylethanolamine (DOPE) for DNA Transfection Cooperating with a Cationic Lipid Bearing Ethylenediamine. Biochimica et Biophysica Acta, 1828, 412-428.

[19] Zuhorn, I.S., Bakowsky, U., Polushkin, E., Visser, W.H., Stuart, M.C., Engberts, J.B. and Hoekstra, D. (2005) Nonbilayer Phase of Lipoplex-Membrane Mixture Determines Endosomal Escape of Genetic Cargo and Transfection Efficiency. Molecular Therapy, 11, 801-810.

Submit or recommend next manuscript to SCIRP and we will provide best service for you:

Accepting pre-submission inquiries through Email, Facebook, LinkedIn, Twitter, etc. A wide selection of journals (inclusive of 9 subjects, more than 200 journals)

Providing 24-hour high-quality service

User-friendly online submission system

Fair and swift peer-review system

Efficient typesetting and proofreading procedure

Display of the result of downloads and visits, as well as the number of cited articles

Maximum dissemination of your research work

Submit your manuscript at: http://papersubmission.scirp.org/

Or contact pp@scirp.org 